\title{
Epidural anesthesia and cancer recurrence rates after radical prostatectomy
}

\section{Anesthésie péridurale et taux de récidive du cancer après une prostatectomie radicale}

\author{
Ban C. H. Tsui, MD • Saifudin Rashiq, MB - Donald Schopflocher, PhD • \\ Albert Murtha, MD • Sunita Broemling, MD · Jennifer Pillay, BSc • \\ Brendan T. Finucane, MB
}

Received: 19 September 2008/Revised: 31 August 2009/Accepted: 1 September 2009/Published online: 13 November 2009

(C) Canadian Anesthesiologists' Society 2009

\begin{abstract}
Purpose To determine the effect of adjunctive epidural local anesthetic and opioid infusion on disease recurrence following radical prostatectomy for adenocarcinoma under general anesthesia.

Methods This article describes a secondary analysis of subjects undergoing radical prostatectomy who had participated previously in a randomized controlled trial evaluating pain control, blood loss, and the need for perioperative allogeneic blood transfusion. The patients were randomly allocated to receive either general anesthesia alone (control group; $n=50$ ) or combined general/ epidural anesthesia (study group; $n=49$ ). A long-term follow-up chart review was undertaken to determine clinically evident or biochemical (Prostate Specific Antigen $>0.2 \mathrm{ng} \cdot \mathrm{mL}^{-1}$ ) recurrence of prostate cancer. Comparison by group was undertaken using survival analysis.
\end{abstract}

This article is accompanied by an editorial. Please see Can J Anesth 2010; 57: 2 .

B. C. H. Tsui, MD $(\bowtie) \cdot$ S. Rashiq, MB · S. Broemling, MD . J. Pillay, BSc · B. T. Finucane, MB

Department of Anesthesiology and Pain Medicine, University of Alberta, 8-120 Clinical Sciences Building, Edmonton, AB T6G 2G3, Canada

e-mail: btsui@ualberta.ca

\author{
A. Murtha, MD \\ Department of Oncology, University of Alberta, \\ Edmonton, AB, Canada \\ D. Schopflocher, PhD \\ Faculty of Nursing, University of Alberta, \\ Edmonton, AB, Canada
}

Results Median disease-free survival for the study as a whole was 1644 days, and the longest recorded survival was 3403 days. Biochemical recurrence of prostate cancer was observed in 11/49 study subjects and 17/50 control subjects. There was one death from prostate cancer in each group and a total of five deaths in the study group and six deaths in the control group. The hazard ratio for recurrence in the study group compared with the control group was 1.33 (95\% confidence intervals $0.64-2.77 ; P=0.44$ by log-rank test).

Conclusion No difference was observed between the epidural and control groups in disease-free survival at a median follow-up time of 4.5 years. There is a need for large randomized controlled trials to determine the ability of epidural analgesia to alter disease recurrence rates following radical prostatectomy.

\section{Résumé}

Objectif Déterminer l'effet d'une thérapie adjuvante d'anesthésique local péridural et perfusion d'opiö̈es sur la récurrence de la maladie après une prostatectomie radicale pour adénocarcinome sous anesthésie générale. Méthode Cet article décrit une analyse secondaire de patients ayant subi une prostatectomie radicale, lesquels avaient précédemment participé à une étude randomisée contrôlée évaluant le contrôle de la douleur, les pertes sanguines et le besoin de transfusions sanguines allogènes en période périopératoire. Les patients ont été randomisés à recevoir soit une anesthésie générale seule (groupe témoin; $n=50$ ) ou une combinaison d'anesthésie générale et péridurale (groupe à l'étude; $n=49$ ). Une révision des dossiers de suivi à long terme a été entreprise afin de 
déterminer la récurrence cliniquement évidente ou biochimique (antigène prostatique spécifique $>0,2 \mathrm{ng} \cdot \mathrm{mL}^{-1}$ ) du cancer de la prostate. La comparaison par groupe a été effectuée par une analyse de la survie.

Résultats La survie médiane sans maladie pour l'ensemble de l'étude était de 1644 jours, et la survie la plus longue enregistrée était de 3403 jours. Une récidive biochimique du cancer de la prostate a été observée chez $11 / 49$ des patients du groupe à l'étude et 17/50 des patients du groupe témoin. Il y a eu un décès dî au cancer de la prostate dans chaque groupe et un total de cinq décès dans le groupe à l'étude et six dans le groupe témoin. Le taux de risque de récurrence était de 1,33 dans le groupe à l'étude par rapport au groupe témoin (95\% intervalle de confiance 0,64, 2,77; $P=0,44$ par test de Mantel-Haenszel). Conclusion Aucune différence n'a été observée entre les groupes péridurale et témoin du point de vue de la survie sans maladie à un suivi médian de 4,5 ans. Des études randomisées contrôlées d'envergure sont nécessaires afin de déterminer la capacité de l'analgésie péridurale à altérer les taux de récurrence de la maladie après une prostatectomie radicale.

The hypothesis that regional anesthesia for cancer surgery might be associated with a decreased risk of disease recurrence is both clinically appealing and biologically plausible. Immunosuppression is likely a contributing factor to cancer recurrence, ${ }^{1}$ and regional anesthesia is known to positively modulate immunity. ${ }^{2}$ This, in turn, may be attributable to the reduction in surgical stress response that regional anesthesia provides by blocking nociceptive afferent inputs. ${ }^{3}$ Allogeneic blood transfusion is known to decrease cancer survival, ${ }^{4,5}$ and regional anesthesia reduces the risk of such transfusions. Our group previously demonstrated that the use of a combined general/epidural hypotensive anesthesia technique during radical prostate surgery resulted in less need for allogeneic blood. ${ }^{6}$ Others have obtained similar results. ${ }^{7,8}$

Five studies exist where the association between type of anesthesia and cancer survival in vivo was examined. All of the studies are retrospective observational investigations that used statistical techniques to control as far as possible for differences in subject and tumor characteristics between treatment arms. In one such study, survival after resection of cutaneous melanoma was found to be better after local infiltration anesthesia than after general anesthesia, ${ }^{9}$ but in two other studies, it was found to be no different. ${ }^{10,11}$ At 3 years postoperatively, paravertebral local anesthetic block combined with general anesthesia for breast cancer surgery was shown to be associated with a mean diseasefree increase in survival of $77-94 \% .^{12}$ Most recently, an analysis of 225 men who underwent open radical prostatectomy for cancer demonstrated a significant association between the use of epidural anesthesia and disease-free survival (hazard ratio for epidural group 0.43) after correcting for a number of known risk factors for recurrence. ${ }^{13}$ The authors of these studies all recognized the potential drawbacks of using retrospective data for this purpose and argued that randomized trials should be conducted. Such trials are underway but far from completion. ${ }^{14}$

In the meantime, we examined the disease-free survival of subjects who participated in a previous randomized trial of combined general/epidural anesthesia for radical prostatectomy. Our specific objectives were to determine the association, if any, between the use of epidural anesthesia and disease-free survival. In the event no association was found, we used serum prostate-specific antigen (PSA) measurements as a marker for prostate cancer recurrence to estimate the smallest survival difference that our dataset could detect.

\section{Methods}

The Health Research Ethics Board of the University of Alberta approved the review of the medical charts of 99 patients who had participated in a randomized trial in 2000 $2001^{6}$ and waived the necessity for specific informed consent from each participant for this secondary analysis. In the previous investigation, the subjects underwent radical prostatectomy for adenocarcinoma by random allocation using either general anesthesia alone (control group; $n=50$ ) or combined general/epidural anesthesia (study group; $n=$ 49). Following induction of general anesthesia, the study group received a loading dose of $0.5 \%$ ropivacaine via thoracic/lumbar epidural catheter to attain a mean arterial pressure from $55-60 \mathrm{mmHg}$. This was followed by a continuous epidural infusion of $0.2 \%$ ropivacaine with fentanyl $2 \mu \mathrm{g} \cdot \mathrm{mL}^{-1}$ to maintain the mean arterial pressure at that level. The control group received intravenous morphine sulphate $10-20 \mathrm{mg}$ for intraoperative analgesia. No arrangements were made for long-term supervision after the study, and no protocol was established for treating the patients following their discharge from hospital.

All operations occurred at least five years before we began the current analysis. We examined hospital charts and records from surgeons' offices to determine the last date each patient was known to be alive or the date and/or cause of death, if applicable. (As a matter of administrative policy, access to the comprehensive computerized database of laboratory test results maintained by our health authority is not permitted for research purposes.) We recorded clinically evident recurrences of prostate cancer and obtained all available postoperative PSA measurements. In accordance with the literature, we defined biochemical recurrence of prostate cancer as the detection of a PSA level $>0.2 \mathrm{ng} \cdot \mathrm{mL}^{-1} \cdot{ }^{15}$ 
We compared survival in the two treatment arms using survival analysis by the life table method (PROC LIFETEST in SAS for Windows version 8.2), and we used a proportional hazards regression model (PROC PHREG) to compare the hazard ratio and $95 \%$ confidence interval for recurrence between the study group and the control group. Since the subjects had been randomly allocated to anesthesia type, we did not apply adjustments for confounding. Subjects with no recurrence of prostate cancer were rightcensored at the last known date they were known to be alive and disease-free, or on the day they died from nonrelated causes. If no postoperative PSA data were available, the subjects were effectively removed from the analysis by right-censoring on the day of hospital discharge. Treatment allocation was temporarily removed from the dataset during the censoring process in an attempt to make it as non-informative as possible.

\section{Results}

Table 1 presents the summary descriptive data of the two groups. The randomization process yielded groups that were well-balanced in all clinically important respects. In the original investigation, 102 subjects were randomized, 51 to each of the study and control groups. However, it was not possible to site the epidural catheter in two patients in the epidural group, and one patient with renal failure was recruited to the control group in violation of protocol. No further data were collected on these subjects; as a result, 50 control group subjects and 49 study group subjects completed the protocol. All subjects survived to hospital discharge. In 22 cases (14 patients in the study group and eight patients in the control group) PSA data were unavailable after hospital discharge. Biochemical recurrence of prostate cancer was observed in 11/49 study group subjects and 17/50 control group subjects. There was one death from prostate cancer in each group. No prostate cancer recurrences presented other than biochemically or by death. There were four other deaths in the study group, all from cancer, one each of colon, kidney, liver, and lung cancer. There were five other deaths in the control group; two from coronary artery disease, one homicide, one from pancreatic cancer, and one from bowel cancer. Progress of the subjects through the investigation is summarized in Fig. 1.

Median survival (to censoring or recurrence) for the study as a whole was 1644 days, and the longest recorded survival was 3403 days. Disease-free survival over time for the two groups is shown in Fig. 2. Log-rank testing indicated that there was no statistically significant difference between strata $(P=0.44)$. The hazard ratio for the study group was 1.33 with wide confidence intervals (0.64-2.77).

\section{Discussion}

This secondary analysis of data from a previous randomized trial showed no advantage in preventing biochemical recurrence of prostate cancer resulting from the adjunctive use of epidural analgesia at the time of radical prostatectomy. This finding implies that either the study was underpowered to detect any real difference or no treatment effect exists.

We used the log-rank test to compare survival between groups. Given our sample size, which was determined by the size of the original trial, a post hoc calculation using an alpha error of 0.05 , a beta error of 0.2 , and the control group's non-recurrence rate of 0.64 indicated that a hazard ratio of at least 0.25 in favour of the epidural group needed to be present to facilitate detection. Indeed, that nonrecurrence rate is an underestimate because it includes subjects censored for lack of follow-up information, some of whom may have experienced a recurrence. Nonetheless,
Table 1 Subject demographics, preoperative prognostic indices, and pathologic tumour staging by treatment allocation

PSA prostate specific antigen

\begin{tabular}{lll}
\hline & Epidural $(n=49)$ & Control $(n=50)$ \\
\hline Age $(\mathrm{yr})$ mean $\pm \mathrm{SD}$ & $63.0 \pm 5.5$ & $63.9 \pm 6.1$ \\
Body mass index $\left(\mathrm{kg} \cdot \mathrm{m}^{-2}\right)$ mean $\pm \mathrm{SD}$ & $28.6 \pm 3.6$ & $27.7 \pm 3.1$ \\
Preoperative serum PSA $\left(\mathrm{ng} \cdot \mathrm{mL}^{-1}\right)$ mean $\pm \mathrm{SD}$ & $10.5 \pm 8.2$ & $8.5 \pm 4.8$ \\
Preoperative Gleason score mean $(95 \% \mathrm{CI})$ & $6(5-8)$ & $6(4-9)$ \\
$\leq 6$ & 31 & 32 \\
7 & 17 & 13 \\
$8-10$ & 2 & 4 \\
Preoperative clinical T stage mode (range) & $\mathrm{T} 2 \mathrm{a}(\mathrm{T} 1 \mathrm{c}-\mathrm{T} 2 \mathrm{~b})$ & $\mathrm{T} 2 \mathrm{a}(\mathrm{T} 1 \mathrm{c}-\mathrm{T} 2 \mathrm{~b})$ \\
Nodal involvement & 1 & 1 \\
Capsular invasion & 18 & 23 \\
Positive margins & 20 & 20 \\
Seminal vesicle involvement & 3 & 5
\end{tabular}




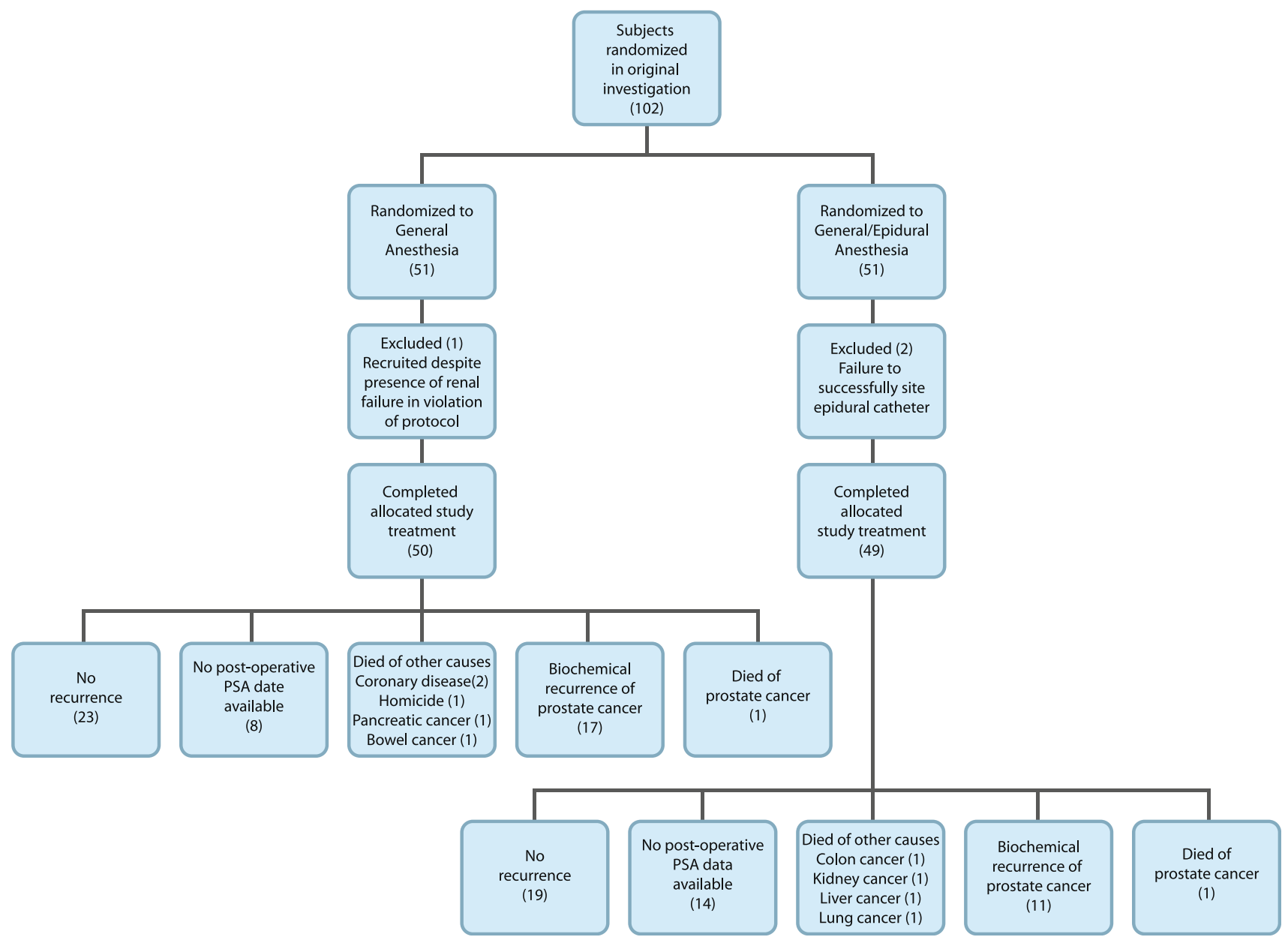

Fig. 1 Progress of subjects through trial

the difference in our epidural group's non-recurrence was much smaller and in the opposite direction. Therefore, our principal explanation as to why we did not obtain a positive result is due to the relatively small size of any potential survival advantage (or disadvantage) conferred by epidural anesthesia compared with the statistical power of the investigation. From a pragmatic standpoint, it could be argued that any intervention that could not deliver an observable improvement over more than 200 subject-years of observation is likely to be of limited clinical value.

It should be noted that biochemical recurrence of disease following prostatectomy is not synonymous with the early detection of clinically significant prostate cancer. About $35 \%$ of patients demonstrate rising PSA; however, the median interval between biochemical recurrence and metastasis is 8 years, and the median interval between metastasis and death is 5 years. ${ }^{16}$ Many patients, especially those of advanced age, may therefore opt to forgo the adverse effects of active treatment for cancer recurrence. This further increases the difficulty of demonstrating that a measure, such as regional anesthesia, taken at the time of surgery can materially affect survival. Our observation period was simply not long enough to yield informative data about prostate cancer deaths.

There is no universally accepted standard for the definition of biochemical recurrence of prostate cancer. Various PSA values between 0.1 and $0.5 \mathrm{ng} \cdot \mathrm{mL}^{-1}$ have been suggested as appropriate cut-points for the definition of recurrent disease, while others have suggested that rising PSA over time is more informative than a single abnormal measurement. ${ }^{16}$ We used $0.2 \mathrm{ng} \cdot \mathrm{mL}^{-1}$, which is supported by the literature ${ }^{15}$ and is the working definition used by our clinicians. Had we used a cut-point of $0.4 \mathrm{ng} \cdot \mathrm{mL}^{-1}$, another well-supported choice, ${ }^{16}$ one study group subject and one control group subject would have been deemed not to have experienced recurrence. The survival period of a further ten study subjects would have been extended by a mean of 0.325 years, and the survival of a further seven control subjects would have been extended by a mean of 0.57 years. In the aggregate, this would not have changed our result. The use of a trend rather than a single value biases the estimation of survival, since the more often PSA is measured 
Fig. 2 Kaplan-Meier survival curves for epidural and control groups

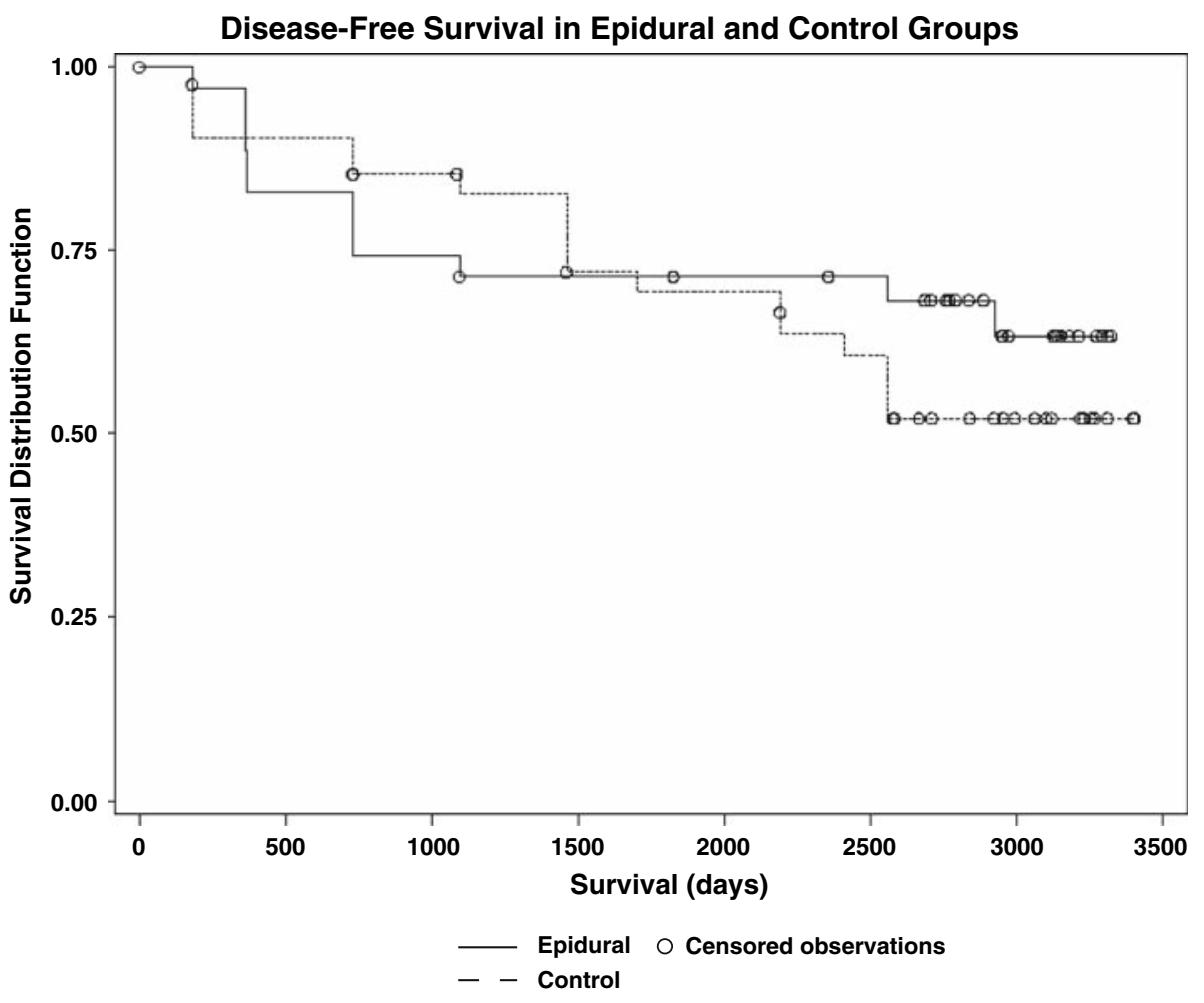

the more rapidly a rise will be detected. In our study, PSA was measured at 3-month intervals for the first year and then annually. Thus, it is difficult to apply to our sample PSA trend criteria from studies where frequent PSA measurements were continued for a longer period after prostatectomy.

An alternative explanation for the difference between our result and that observed by Biki et al. ${ }^{13}$ (the retrospective study which found that the use of epidural anesthesia conferred a significant survival advantage in prostatectomy) is the relative absence of selection bias in our study. Despite every effort on their part to correct for confounding factors, they would have been unable to correct for biases that were not recorded or were unknown. Clinical experience suggests to us that the decision to offer or withhold epidural analgesic supplementation during prostatectomy is not independent of the anticipated difficulty of the anesthetic component or, by inference, the surgical component of the procedure. An obese person, for example, might present greater challenges in terms of both successful placement of an epidural catheter and adequate surgical disease clearance, and these data were not recorded in the retrospective investigation. At the same time, we acknowledge that we violated the intention-to-treat principle to a small extent by failing to obtain long-term follow-up data on the three subjects who were randomized but excluded. We did not have sufficient information on those individuals to include them retrospectively. We hypothesize that both factors leading to these exclusions (inability to site the epidural catheter in the two study group recruits and renal failure in the control recruit) could only have worsened and not improved their chances of survival. Accordingly, the most extreme bias that these omissions could have introduced, which would not have changed our study outcome, would have been the net removal of a single subject from the study group at increased risk of recurrence.

The value of this study is that it emphasizes the fact that the debate about the use of regional anesthesia for the specific purpose of cancer recurrence prevention can only be meaningfully informed by much larger prospective trials. Unfortunately, while there are ongoing ambitious and wellpowered trials regarding the preventative effect of regional anesthesia on the recurrence of breast ${ }^{17}$ and colorectal ${ }^{18}$ cancer, they are not projected to be completed before 2013 and 2022, respectively. A planned study of epidural anesthesia on endometrial cancer recurrence rates was recently abandoned because of a lack of an adequate study population, ${ }^{19}$ which may exemplify some of the difficulties involved in conducting this kind of investigation. Biki's retrospective analysis gave us a hazard ratio of 0.43 in favour of epidural anesthesia. ${ }^{11}$ In order to demonstrate this survival advantage in a randomized trial, assuming a 5-year control biochemical recurrence rate of $32 \%$, a 5-year follow-up on 210 subjects would be required. That figure increases to 3064 in order to detect the smallest treatment effect contained in the $95 \%$ confidence bounds of Biki's result (0.83). 
Ultimately, the conduct of a prospective trial of sufficient power and duration to provide definitive answers to these questions may prove to be too difficult and costly, in which case, the best available data from meta-analyses or population studies will have to suffice. We caution against drawing conclusions from retrospective studies that do not contain at least sufficient information about the manner in which the decision whether to offer regional anesthesia was made. Nevertheless, if the estimates of improved cancer survival attributed to the use of regional anesthesia derived from the retrospective studies are even approximately correct, every effort should be made to demonstrate them prospectively, since this would constitute a very substantial improvement in outcome. This would be of crucial importance for patients, anesthesiologists, and surgeons alike. The benefits of epidural anesthesia during radical prostatectomy are proven in terms of less blood loss and less need for allogeneic blood transfusion and pain control. For the time being, we suggest that the question whether epidural anesthesia also confers improvement in prostate cancer recurrence rates continues to be regarded as unanswered.

Acknowledgments We are indebted to members of the Department of Urology at the University of Alberta for their invaluable insights, Drs. Paul O'Connor and John Hanson for their contributions to the original trial, and to Dr. Timur Özelsel for translating one of the references into English.

Funding source Supported in part by a Clinical Scholar Award, Alberta Heritage Foundation for Medical Research, Alberta, Canada.

Conflicts of interest None declared.

\section{References}

1. Kelbel I, Weiss M. Anaesthetics and immune function. Curr Opin Anaesthesiol 2001; 14: 685-91.

2. Whelan P, Morris PJ. Immunological responsiveness after transurethral resection of the prostate: general versus spinal anaesthetic. Clin Exp Immunol 1982; 48: 611-8.

3. Rodgers A, Walker N, Schug $S$, et al. Reduction of postoperative mortality, morbidity with epidural or spinal anaesthesia: results from overview of randomised trials. BMJ 2000; 321: 1493.

4. Heal JM, Chuang C, Blumberg N. Perioperative blood transfusions and prostate cancer recurrence and survival. Am J Surg 1988; 156 : 374-80.
5. McClinton S, Moffat LE, Scott S, Urbaniak SJ, Kerridge DF. Blood transfusion and survival following surgery for prostatic carcinoma. Br J Surg 1990; 77: 140-2.

6. O'Connor PJ, Hanson J, Finucane BT. Induced hypotension with epidural/general anesthesia reduces transfusion in radical prostate surgery. Can J Anesth 2006; 53: 873-80.

7. Frank E, Sood OP, Torjman M, Mulholland SG, Gomella LG. Postoperative epidural analgesia following radical retropubic prostatectomy: outcome assessment. J Surg Oncol 1998; 67: 117-20.

8. Hendolin H, Alhava E. Effect of epidural versus general anaesthesia on perioperative blood loss during retropubic prostatectomy. Int Urol Nephrol 1982; 14: 399-405.

9. Schlagenhauff $B$, Ellwanger $U$, Breuninger $H$, Stroebel $W$, Rassner $G$, Garbe $C$. Prognostic impact of the type of anaesthesia used during the excision of primary cutaneous melanoma. Melanoma Res 2000; 10: 165-9.

10. Melchi CF, Mele A, Baliva $G$, et al. Prognostic value of anesthesia type for patients treated for cutaneous melanoma. Dermatol Surg 1995; 21: 786-8.

11. Seebacher C, Heubaum F, Kuster P, Steinert W, Koch R. Comparative analysis of narcosis and local anesthesia in surgery of malignant melanoma of the skin (German). Hautarzt 1990; 41: 137-41.

12. Exadaktylos AK, Buggy DJ, Moriarty DC, Mascha E, Sessler DI. Can anesthetic technique for primary breast cancer surgery affect recurrence or metastasis? Anesthesiology 2006; 105: 660-4.

13. Biki B, Mascha E, Moriarty DC, Fitzpatrick JM, Sessler DI, Buggy $D J$. Anesthetic technique for radical prostatectomy surgery affects cancer recurrence: a retrospective analysis. Anesthesiology 2008; 109: 180-7.

14. Sessler DI. Does regional analgesia reduce the risk of cancer recurrence? A hypothesis. Eur J Cancer Prev 2008; 17: 269-72.

15. Freedland SJ, Sutter ME, Dorey F, Aronson WJ. Defining the ideal cutpoint for determining PSA recurrence after radical prostatectomy. Prostate-specific antigen. Urology 2003; 61: 365-9.

16. Freedland SJ, Humphreys EB, Mangold LA, et al. Risk of prostate cancer-specific mortality following biochemical recurrence after radical prostatectomy. JAMA 2005; 294: 433-9.

17. ClinicalTrials.gov [Internet]. Bethesda, MD: National Institutes of Health. 1993. Trial no. NCT00418457, Regional anesthesia and breast cancer recurrence. http://www.clinicaltrials.gov/ct2/show/ NCT00418457?term $=$ NCT $+00418457 \&$ rank=1. Accessed May 2009.

18. ClinicalTrials.gov [Internet]. Bethesda, MD: National Institutes of Health. 1993. Trial no. NCT00684229, Regional anesthesia in colon rectal cancer. http://www.clinicaltrials.gov/ct2/results?term= NCT00684229. Accessed May 2009.

19. ClinicalTrials.gov [Internet]. Bethesda, MD: National Institutes of Health. 1993. Trial no. NCT00531349, Regional anesthesia and endometrial cancer recurrence. http://www.clinicaltrials.gov/ ct2/results?term=NCT00531349. Accessed May 2009. 